\title{
9 Kostensensible Leitlinien: Einschätzungen von Entscheidungsträgern und Interessenvertretern im deutschen Gesundheitswesen
}

Swantje Reimann, Daniel Strech und Georg Marckmann

\subsection{Hintergrund und Ziel der Studie}

Aufgrund steigender Gesundheitsausgaben durch die Alterung der Bevölkerung (Peters et al. 2010) und medizinisch-technische Innovationen wird sich die finanzielle Situation der deutschen Gesetzlichen Krankenversicherung (GKV) nach weithin geteilter Auffassung zukünftig weiter verschärfen (Marckmann 2007; Zentrale Kommission zur Wahrung ethischer Crundsätze in der Medizin und ihren Grenzgebieten [Zentrale Ethikkommission] bei der Bundesärztekammer 2007). Dem gegenüber steht eine sinkende Zahl von Versicherten, die dieses System mit ihren Beiträgen finanzieren, sodass den steigenden Ausgaben begrenzte oder sogar sinkende Einnahmen gegenüberstehen.

Dass Ärzte unter einem Handlungsdruck stehen, das begrenzte Budget eines Krankenhauses durch Diagnostik und Therapie nicht über die Maßen zu strapazieren und Patienten medizinisch sinnvolle Leistungen vorzuenthalten, konnte in verschiedenen Studien gezeigt werden (vgl. u.a. Boldt u. Schöllhorn 2008; Kern et al. 1999). So auch in dem vom BMBF finanzierten interdisziplinären Forschungsverbund „Allokation“. Innerhalb dieses Projektes wurde eine repräsentative Fragebogenerhebung zum Ausmaß impliziter Rationierung durchgeführt. Die Ergebnisse zeigen, dass mehr als drei Viertel (78\%) der antwortenden Ärzte im letzten halben Jahr mindestens einmal nützliche Leistungen aus Kostengründen vorenthalten hatten. 13 Prozent gaben an, dies min- 
9 Kostensensible Leitlinien: Einschätzungen von Entscheidungsträgern und Interessenvertretern im deutschen Gesundheitswesen

destens einmal in der Woche zu tun (Strech et al. 2009a) (s. Kap. 1). Demnach ist implizite Rationierung im deutschen Gesundheitswesen kein singuläres Phänomen mehr, sondern eine verbreitete Option, mit begrenzten Leistungen in der Gesundheitsversorgung umzugehen. Nach wie vor fühlen sich Ärzte aber vor allem ihrem jeweils individuellen Patienten verpflichtet: 83\% der Befragten gaben an, ihre Aufgabe sei die bestmögliche Versorgung der Patienten unabhängig von den dadurch entstehenden Kosten (Strech et al. 2009a). Implizite Leistungsbegrenzungen belasten nicht nur die ärztlichen Entscheidungsträger und das Arzt-Patient-Verhältnis, sondern bergen darüber hinaus die Gefahr, dass medizinische Leistungen nach intransparenten, von Fall zu Fall wechselnden und ethisch häufig nicht gut begründeten Kriterien verteilt werden (Marckmann 2007; Strech et al. 2008a).

Explizite Leistungsbegrenzungen können demgegenüber Ärzte in solchen Entscheidungssituationen entlasten. Sie erlauben transparente Allokationsentscheidungen nach klar definierten, für alle Patienten gleichermaßen verbindlichen Kriterien (Marckmann 2007). Dies sichert nicht nur die Gleichbehandlung der Patienten, sondern erlaubt darüber hinaus eine evidenzbasierte Abwägung von Kosten und Qualität der Versorgung. Im Forschungsverbund „Allokation“ wurden mit den Kostensensiblen Leitlinien (KSLL) Instrumente entwickelt, um die letztlich unvermeidbaren Rationierungen in einer ethisch vertretbaren und ökonomisch sinnvollen Art und Weise durchzuführen (vgl. hierzu ausführlicher Kap. 2 u. 3). Für zwei medizinische Maßnahmen - Medikamentefreisetzende Stents (drug eluting stents, DES) und implantierbare Kardioverter-Defibrillatoren (ICDs) - wurden exemplarische KSLL erstellt, um einen Einblick in die methodischen Herausforderungen bei der Erstellung der KSLL zu gewinnen (s. Kap. 4 u. 5). Eine empirische Evaluierung der KSLL im Bereich der GKV-Versorgung ist nicht möglich, da diese KSLL den Versorgungsanspruch der Versicherten in einer unter den gegebenen Rahmenbedingungen rechtlich nicht zugelassenen Art und Weise einschränken würden (s. Kap. 8). Um dennoch eine Einschätzung der Stärken und Schwächen sowie der Akzeptanz expliziter Leistungsbegrenzungen mittels KSLL zu gewinnen, haben wir mit Entscheidungsträgern und Vertretern unterschiedlicher Interessensgruppen aus dem deutschen Gesundheitswesen Experteninterviews geführt. Als Gesprächsgrundlage dienten die beiden exemplarischen Kostensensiblen Leitlinien und eine kurze Einführung in die KSLL. Im vorliegenden Beitrag berichten wir über die Ergebnisse dieser Interviewstudie.

\subsection{Kostensensible Leitlinien als Instrument expliziter Rationierung}

Kostensensible Leitlinien (KSLL) stellen ein Instrument zur Umsetzung expliziter Rationierung in der Versorgungspraxis dar. Ihnen liegt folgende ethische Überlegung zugrunde (vgl. hierzu ausführlicher Kap. 2 den Beitrag von Marckmann in diesem Band): Wenn Leistungseinschränkungen unvermeidlich 
sind, sollten diese so durchgeführt werden, dass den Patienten ein möglichst geringer (Zusatz-)Nutzen vorenthalten wird. Ausgeschlossen werden demnach Maßnahmen, die - im Vergleich zu kostengünstigeren Alternativen - unter Aufwendung sehr hoher Kosten einen nur geringen zusätzlichen Nutzen für die Patienten bieten. Bei der Erstellung von KSLL sind deshalb zunächst auf der Grundlage der verfügbaren wissenschaftlichen Evidenz zu Nutzen- und Schadenspotenzial der Maßnahmen Subgruppen von Patienten zu identifizieren, die mehr oder weniger von bestimmten Maßnahmen profitieren. Mit einer KSLL würde dann der Einsatzbereich einer medizinischen Intervention auf diejenige Patientensubgruppe beschränkt, bei der ein höherer Nutzengewinn zu erwarten ist, während die anderen Patienten, die kaum von der Intervention profitieren, auf alternative Maßnahmen ausweichen müssen, sofern diese verfügbar sind. Wo die Grenze zwischen den beiden Cruppen gezogen wird, hängt wesentlich davon ab, welches Verhältnis von Nutzen und Kosten gerade noch als akzeptabel angesehen wird.

\subsection{Methodik}

Um Einschätzungen zu den Stärken und Schwächen sowie der Akzeptanz der KSLL zu ermitteln, führten wir fokussierte, leitfadengestützte Experteninterviews durch. Dieses (semi-)qualitative Verfahren bietet die Möglichkeit, dem jeweilig Befragten ausreichend Raum zu geben, eigene relevante Überlegungen, Deutungen und Themen im Hinblick auf den Stimulus zu entwickeln und mitzuteilen. Den Fokus der Interviews bildeten die beiden exemplarischen KSLL zu den Medikamente-freisetzenden Stents (DES) und den implantierbaren Kardioverter-Defibrillatoren (ICD). Der von uns eingesetzte Leitfaden basierte auf den Ergebnissen der vorangegangenen Untersuchungen und rahmte die Gespräche (vgl. folgende Aufzählung).

\section{Leitfaden Experteninterview zu KSLL}

- Zu Beginn möchten wir Sie bitten, uns Ihren ersten Eindruck zu schildern, den Sie beim Lesen der KSLL hatten. Erzählen Sie doch bitte, was Ihnen zuerst aufgefallen ist.

- Gibt es Aspekte, die Ihnen zu kurz kommen/die Ihnen dabei nicht so wichtig erscheinen?

- Gibt es Neues, was Sie vorher in dieser Art und Weise noch nicht gesehen haben?

- Können Sie sich vorstellen, dass diese KSLL einen Beitrag zur Lösung relevanter Probleme im Gesundheitswesen leisten könnten? (Wenn ja, inwiefern? Wenn nein, welche Gründe sprechen dagegen?)

- Wären KSLL in Ihrem Arbeitsbereich geeignete/anwendbare Instrumente?

- Welche Erleichterungen/Belastungen könnte ein Einsatz der KSLL zur Folge haben?

- Was würde die Akzeptanz der KSLL Ihrer Meinung nach beeinflussen? 
9 Kostensensible Leitlinien: Einschätzungen von Entscheidungsträgern und Interessenvertretern im deutschen Gesundheitswesen

nas würde die Praktikabilität der KSLL Ihrer Meinung nach beeinflussen?

- Wie schätzen Sie die Verbindlichkeit solcher KSLL ein? Nach welchen Kriterien sollten gegebenenfalls Ausnahmen möglich sein?

- Könnten KSLL Richtliniencharakter haben?

- Wer sollte KSLL für das deutsche Gesundheitswesen erstellen?

- Möchten Sie noch etwas ergänzen?

Für das Interviewsample wurden zunächst relevante Institutionen des deutschen Gesundheitswesens identifiziert. Anschließend wurden Vertreter der Selbstverwaltung, der Kostenträger und Leistungserbringer, von Verbraucherschutz und Politik postalisch kontaktiert, über das Projekt informiert und um Teilnahme an einem Interview gebeten. Dieses purposive sampling wurde mit dem Schneeballprinzip gekoppelt, um unterschiedliche Experten für eine Teilnahme zu gewinnen. Durch beide Suchstrategien sollte ein möglichst breites Untersuchungsfeld mit wichtigen Akteuren und Institutionen abgebildet werden. Eine Zusammenstellung der Interviewpartner findet sich in Tabelle 32.

Mit den interessierten Experten wurden Termine an ihrem jeweiligen Arbeitsplatz vereinbart; sie bekamen im Vorfeld die beiden KSLL sowie allgemeine Hintergrundinformationen zu den KSLL zugesendet. Anonymität der Daten und deren ausschließliche Verwendung in diesem Projekt wurde zugesichert. Das Einverständnis der Interviewpartner wurde schriftlich festgehalten. Die Interviews wurden von März bis Juni 2009 von mindestens einer Mitarbeiterin (SR) durchgeführt, in einigen Fällen auch ergänzt durch weitere Mitarbeiter des Forschungsverbundes (GM, DS, DF). Die Gespräche wurden aufgezeichnet und vollständig transkribiert.

\section{Tab. 32 Untersuchungs-Sample Interessenvertreter im deutschen Gesundheitswesen}

\begin{tabular}{lll}
\hline Interessenvertreter & Institution & TN \\
\hline \multirow{2}{*}{ Kostenträger } & Spitzenverband der Gesetzlichen Krankenversicherungen (GKV) & 1 \\
\hline & Bundesverband der Allg. Ortskrankenkasse (AOK-BV) & 1 \\
\hline & Bundesärztekammer (BÄK) & 1 \\
\cline { 2 - 3 } & Kassenärztliche Bundesvereinigung (KBV) & 1 \\
\hline Selbstverwaltung & Kediologen & 6 \\
\hline & Medizinischer Dienst des Spitzenverbandes (MDS) & 4 \\
\hline & $\begin{array}{l}\text { Institut für Qualität und Wirtschaftlichkeit im Gesundheitswesen } \\
\text { (IQWiG) }\end{array}$ & 1 \\
\hline & Gemeinsamer Bundesausschuss (G-BA) & 1 \\
\hline Politik & Fraktionsmitglied Parlamentspartei & 2 \\
\hline Betroffene & Verbraucherzentrale (vzbv) & 1 \\
\hline
\end{tabular}


Die Auswertung erfolgte nach der Inhaltsanalytischen Methode (Mayring 2008), wobei die Anmerkungen, Hinweise und Darstellungen der Interviewpartner in einem Klassifikationssystem mit Ober- und Unterkategorien zusammengefasst wurden. Die Erstellung der deskriptiven Kategorien erfolgte einerseits deduktiv durch die Vorgabe der Leitfaden-Kategorien und zusätzlich induktiv aus den Expertengesprächen.

Die Auswertung wurde schrittweise vorgenommen, wobei im ersten Schritt Aussagen aus acht Interviews zu Oberthemen zusammengefasst wurden. Daraufhin folgte die Untergliederung in die jeweiligen Unterkategorien sowie die Zuordnung von Beispielen. Nachdem ein vorläufiges Kategoriensystem durch eine Mitarbeiterin (SR) erstellt wurde, wurde dieses hinsichtlich der Validität unabhängig durch zwei weitere Mitarbeiter (GM, DS) überprüft, Nichtübereinstimmungen wurden diskutiert, bei Bedarf wurde das Kategoriensystem nochmals geordnet und modifiziert. In einem zweiten Schritt wurden die restlichen sieben Interviews in das Kategoriensystem aufgenommen, um die Gültigkeit des Systems zu überprüfen und eine theoretische Sättigung oder gegebenenfalls Hinweise auf noch notwendige Anpassungen der Kategorien zu erhalten.

Aus dem Bereich der Kostenträger im Gesundheitswesen konnte je ein Interviewpartner des Spitzenverbandes der Gesetzlichen Krankenversicherungen (GKV), des AOK-Bundesverbandes und je zwei Vertreter einer Klinikverwaltung einbezogen werden. Aus dem Bereich der potenziellen Anwender von KSLL, den Leistungserbringern, stellten sich mehrere Kardiologen zu Verfügung, sowie je ein Vertreter der Bundesärztekammer und der Kassenärztlichen Bundesvereinigung. Institutionen wie den Gemeinsame Bundesausschuss (G-BA), das Institut für Qualität und Wirtschaftlichkeit im Gesundheitswesen (IQWiG) und den Medizinische Dienst der Krankenkassen haben wir der Selbstverwaltung zugerechnet. Die Patientensicht wurde durch eine repräsentative Vertreterin des Verbraucherzentrale Bundesverband (vzbv) und die politische Ebene durch Fraktionsmitglieder zweier Parlamentsparteien vertreten.

Das Sample der Interviewpartner bildet u.E. wichtige Interessensgruppen und Akteure im deutschen Gesundheitswesen ab und kann zumindest eine Tendenz wiedergeben, wie die KSLL im deutschen Gesundheitswesen beurteilt werden. Die Annahme der Repräsentativität der Experten für ihren jeweiligen Arbeitsbereich stellt die entscheidende Komponente für die folgenden Ergebnisse dar. Es können durch ein solches Verfahren jedoch keine Aussagen hinsichtlich der Verteilung und der Häufigkeiten getroffen werden.

Insgesamt wurden 15 Interviews mit 20 Teilnehmern durchgeführt. Die Interviewdauer variierte von 30 bis zu 85 Minuten. 
9 Kostensensible Leitlinien: Einschätzungen von Entscheidungsträgern und Interessenvertretern im deutschen Gesundheitswesen

\subsection{Ergebnisse}

In einer Kategorienordnung ersten Grades ist das Kriterium der Zustimmung (positive Einschätzung) oder Ablehnung (negative, skeptische Einschätzung) zur Trennung der insgesamt 773 den Interviews entnommenen Aussagen verwendet worden. Aufgrund dieser relativ groben Zuweisung wurden 499 Aussagen der ablehnenden Haltung und 274 Aussagen einer mehr zustimmenden Haltung zugeordnet. Eine Übersicht der Kategorien ersten und zweiten Grades kann Tabelle 33 entnommen werden.

Nachfolgend werden die Hauptkategorien mit den jeweils dazugehörenden Unterkategorien beschrieben sowie mit Beispielsätzen expliziert.

\subsubsection{Grundannahmen}

Bezüglich der den KSLL zugrunde liegenden Annahmen werden hier kritische Anmerkungen dargestellt, da grundsätzlich zustimmende Einschätzungen in den Kategorien Akzeptanz, Arzt-Patient-Beziehung und Funktion der KSLL verortet angenommen werden.

Schon die Ausgangsbehauptung der KSLL, dass der demografische Wandel eine Ursache für die Kostensteigerung sei, stelle sich als schwierig und auch als gefährlich dar. Im deutschen Gesundheitswesen bestünde vielmehr eine Zurückhaltung gegenüber Modellen, die eine monetäre Betrachtung beinhalten .

„... man wird dann schon in eine Richtung gebahnt durch dieses Blatt Papier, die ...ja wo ich dann gar nicht mehr weiß, was soll ich denn eigentlich jetzt zu Ihren kostensensiblen Leitlinien antworten, wenn ich das ganze Konzept eher fragwürdig finde“ (TN5; 18).

Dieses explizite Instrument würde eine Wertediskussion in Gang setzen, da die Grundannahmen eine Auseinandersetzung mit weltanschaulichen und politischen Entscheidungen provoziere. Eine Gefahr wird hierbei in der bottomup-Einführung des Utilitarismus gesehen, der den für die Gesundheitsversorgung in Deutschland konstitutiven Werten widerspräche. Ebenso sei die Auslegung des Verhältnisses von Nutzen, Notwendigkeit und Wirtschaftlichkeit derzeit noch strittig. Gerade das Einbeziehen von Kosten einer medizinischen Leistung würde eher abschreckend wirken, zumal mit der privatwirtschaftlichen Verfügbarkeit der ausgeschlossenen Leistungen eine Zwei-Klassen-Medizin drohe.

„Also von daher, glaube ich, da Sie ja auch gezeigt haben, dass wir in Deutschland ja sicher nicht die ... an vorderster Front sind da in dem Bereich, sondern wahrscheinlich hier eher die Einstellung bisher ja auch war: jeder kriegt alles was er braucht. Da ist das, denke ich, im ärztlichen Bereich noch eine Schwierigkeit, das auch so umzusetzen"(TN17; 48). 


\subsection{Ergebnisse}

Tab. 33 Ordnung der Ober- und Unterkategorien

\begin{tabular}{|c|c|}
\hline Oberkategorien & Unterkategorien \\
\hline \multirow[t]{3}{*}{ Grundannahmen } & Ausgangsbehauptung \\
\hline & Wertediskussion \\
\hline & alternativlose Darstellung \\
\hline \multirow[t]{9}{*}{ Methodik - Stärken } & Ansatz des Stufenverfahrens \\
\hline & Ausgangspunkt an Sterblichkeit \\
\hline & Orientierung an Wirksamkeit \\
\hline & Identifizierung von Patienten-Subgruppen und spezifischen Indikationsbereichen \\
\hline & wissenschaftliche Fundierung \\
\hline & Verwendung QALY-Konzept \\
\hline & Verwendung von Grenzbereichen/Spannen \\
\hline & Transparenz von Entscheidungsprozessen und Deutlichkeit \\
\hline & Differenzierung zwischen Kosten und Nutzen \\
\hline \multirow{3}{*}{$\begin{array}{l}\text { Methodik - Herausforde- } \\
\text { rungen }\end{array}$} & problematische Datenlage \\
\hline & Verwendung QALY-Konzept \\
\hline & $\begin{array}{l}\text { formale Aspekte (Lesbarkeit, Oberflächlichkeit der Herleitung, fehlender Bezug zu } \\
\text { existierenden Leitlinien) }\end{array}$ \\
\hline \multirow{3}{*}{$\begin{array}{l}\text { Bedingungen zur } \\
\text { Förderung von Akzeptanz }\end{array}$} & Kommunikation an die Patienten \\
\hline & Interessen hinter den Leitlinien \\
\hline & Erhöhung der Qualität \\
\hline \multirow{3}{*}{$\begin{array}{l}\text { Bedingungen, die } \\
\text { Akzeptanz senken }\end{array}$} & politische Rationalitäten \\
\hline & finanzielle Gewinne durch (rat.) Maßnahmen \\
\hline & Betroffenheit aus Patientensicht \\
\hline \multirow[t]{2}{*}{ Praktikabilität } & Anreizsysteme \\
\hline & Format der Leitlinie \\
\hline \multirow[t]{3}{*}{ Erleichterung für den Arzt } & Beendigung des Konkurrenzkampfes \\
\hline & Entlastung von Verantwortung \\
\hline & Haftungsrechtliche Erleichterungen \\
\hline \multirow[t]{2}{*}{ Belastungen für den Arzt } & $\begin{array}{l}\text { Divergenz zwischen beruflichem Ethos und kostensensiblen Beschränkungen (Arzt } \\
\text { als Doppelagent) }\end{array}$ \\
\hline & Verschärfung des Konkurrenzkampfes zwischen Kliniken \\
\hline \multirow[t]{3}{*}{ Verbindlichkeit } & Entscheidungsautonomie bei Arzt \\
\hline & hohe Verbindlichkeit für GKV \\
\hline & entscheidungsbegleitendes Instrument \\
\hline \multirow[t]{3}{*}{ Funktion der KSLL } & rationales Ressourcenmanagement \\
\hline & Grenzziehung Mitteleinsatz GKV \\
\hline & Diskussionsbeitrag \\
\hline \multirow[t]{2}{*}{ Ersteller der Leitlinien } & Konsens \\
\hline & Stufenverfahren \\
\hline
\end{tabular}


9 Kostensensible Leitlinien: Einschätzungen von Entscheidungsträgern und Interessenvertretern im deutschen Gesundheitswesen

Weiterhin wurde die alternativlose Darstellung sowohl der Grundannahmen als auch der Problemlösung kritisiert. Ob sich in Deutschland überhaupt eine Rationierungsfrage und daraus eine Diskussionsnotwendigkeit stellt, sei fraglich und nicht allgemein akzeptiert. Andere Wege, im Gesundheitswesen effizient zu wirtschaften, seien beispielsweise in Form von Strukturänderungen und nicht dem Vorenthalten medizinischer Maßnahmen denkbar.

„,... und ich würde sagen, bevorich also dann Leuten medizinisch wirksame Leistungen vorenthalte, bin ich eigentlich als, sage ich mal, dem Grundgesetz verpflichtet, dem Gemeinwesen eigentlich der Bevölkerung schuldig, dann zunächst mal an die Pfründe der bevorzugten Arztgruppen und an die Strukturen und an die Krankenhäuser dran zu gehen. Also ich persönlich, sage ich mal, halte das für ethisch nicht vertretbar, explizite Rationierung zu machen“(TN14; 26).

\subsubsection{Methodik}

Hinsichtlich der Methodik der KSLL wurde der Ansatz des Stufenverfahrens der Entscheidung positiv hervorgehoben, da es eher zu Zustimmung führe, ebenso der Ausgangspunkt an der Sterblichkeit und die Orientierung an der Wirksamkeit (Outcome). Die Identifizierung von Patienten-Subgruppen und von spezifischen Indikationsbereichen mache das Verfahren nachvollziehbar und sei vorteilhafter gegenüber einem pauschalen Ausschluss bestimmter Leistungen. Der Allokations-Ansatz sei durch eine wissenschaftliche Fundierung und eine Deutlichkeit gekennzeichnet, die sich positiv auf die Akzeptanz eines solchen Verfahrens auswirke. Die angegebenen Grenzbereiche oder Spannen erlaubten eine differenzierte Entscheidung, was einer konkreten, individuellen Behandlung eher förderlich sei als ein pauschaler Grenzwert. Der Bezug zum QALY-Konzept (quality adjusted life years, qualitätskorrigierte Lebensjahre) mache das Verfahren offen, transparent und auch gerecht.

„Also zunächst mal den Ansatz mit den Subgruppen, der ist sicherlich ok, das finde ich ganz gut“ (...) „Aber sozusagen die ... so prinzipielle Spannen anzugeben und so, das ist sicherlich sinnvoll“ (TN3; 2,16).

Die Transparenz der Entscheidungsprozesse bei den beiden KSLL sei ein weiterer methodischer Vorteil, da dies die Nachvollziehbarkeit der Vorgaben erhöhe. Der Ansatz könne der Suche nach einem effizienten Versorgungsmanagement entgegen kommen, da er rational und nicht von Willkür getragen sei. Durch die gewählte kurze und knappe Darstellung könne der Ansatz plausibel gemacht werden und sei verständlich.

„Was mich ja auch positiv überrascht hat, war die kurze, knappe Darstellung“ (TN20;7).

Die Differenzierung zwischen Kosten und Nutzen und dabei vor allem das Einbeziehen und die inhaltliche Nähe zu existierenden Leitlinien sei vorteilhaft für 
die Akzeptanz der KSLL. Im Kern sei der aufgegriffene Gedanke richtig, auf vorhandene Leitlinien aufzubauen und die ökonomische Beurteilung ergänzend hinzuzufügen. Dieses Einbeziehen sei als eine sachliche und differenzierte Gegenüberstellung von Kosten- und Nutzenaspekten deutlich nachvollziehbar und könne als logische Konsequenz aus dem evidenzbasierten Leitliniengedanken folgen.

„Was ich positiv fand ist, dassja da eine sehr differenzierte Abwägung von Kosten und Nutzen vorgesehen ist“"(TN2; 2).

Problematisch angesehen wurde die zugrunde liegende Datenlage. Die Nutzenbewertung, nicht nur der angesprochenen Behandlungen, stünde noch aus und aus diesem Grund sei es voreilig, Kosten einzubeziehen. Die insuffiziente Evidenzlage sei ein entscheidender Limitationsfaktor. Auch sei der Mehrnutzen einer Leitlinie mit zusätzlicher Betrachtung der Kosten fraglich und nicht per se gegeben. Solch ein Vorgehen sei zudem in Deutschland ohne Rechtsgrundlage, auch seien die Stents nicht durch die Kosten-Nutzen-Bewertung nach $\mathbb{3}$ 35b SGB V abgedeckt, da es sich nicht um Arzneimittel handele. Wichtig sei eine standardisierte Ausgangsmethodik, da sich sonst Verdachtsmomente ergeben, diese KSLL könnten hinsichtlich partikulärer Ziele bestimmter Interessensgruppen modelliert werden.

„... bisschen rechts überholt, dass die Diskussion um die Kosten-Nutzen-Bewertung so einen hohen Stellenwert einnimmt, wo wir eigentlich in der alltäglichen Arbeit damit kämpfen, dass die Nutzenbewertung eigentlich noch kaum als wirklich entscheidungsrelevant wahrgenommen wird“ (TN12; 8).

Speziell wurde das QALY-Konzept kritisch hinterfragt, da dies in den KSLL unzureichend erläutert werde und in Deutschland kein akzeptiertes bzw. ein mit vielen Unsicherheiten einher gehendes Konzept sei. Konkrete Unklarheiten bestünden unter anderem hinsichtlich der vorgenommenen Subgruppenanalyse, bezüglich der Konfidenzintervalle der QALYs und der zugrundeliegenden Datenlage. Gleichfalls gäbe es mögliche Interessenkonflikte bei der Definition des cut-off-Wertes, hier könne wiederum eine Zielgruppenorientierung nicht ausgeschlossen werden.

,... es ist eben bislang glaube ich auch noch kein so ein richtiger Konsens hier auch in Deutschland so diese costs per life year gain, das QALY und was weiß ich dieses das ist noch wenig verankert, (...) da stößt man hier glaube ich zum Teil auch noch auf Unverständnis. Dasist ... das heißt, wenn dieser Konsens nicht da ist, istes schon mal ganz schwierig“(TN15; 84).

Formale Aspekte der Leitlinien bezogen sich einerseits auf die Lesbarkeit der Leitlinie, die nicht gegeben sei, aber auch auf die Oberflächlichkeit der Herleitung, die für die beiden speziellen Fälle zu kurz sei (Anm.: Den Interviewpartnern stand nur die Kurzversion der Leitlinien zur Verfügung, die keine differenzierte Einschätzung der Evidenzgrundlage erlaubt.) Zudem müsse ein 
9 Kostensensible Leitlinien: Einschätzungen von Entscheidungsträgern und Interessenvertretern im deutschen Gesundheitswesen

deutlicherer Bezug zu schon vorhandenen Leitlinien hergestellt werden. Auch sei das Problem der Trennschärfe und der Stabilität der Berechnungsgrundlage nicht gelöst. Unklarheit bestünde auch darüber, ob der Kosten-Effektivitäts-Grenzwert indikationsbezogen oder indikationsübergreifend zu definieren sei. Die unzureichende Validität der Messmethode der Linksventrikulären Ejektionsfraktion (LVEF) bei der ICD-KSLL stelle ein weiteres Problem dar, da es keinen Goldstandard gäbe und die Ergebnisse von vielfältigen, schlecht kontrollierbaren Bedingungen beeinflusst seien. Die Entscheidung von diesem einzelnen Wert abhängig zu machen, sei klinisch kaum haltbar, da der Untersucher durch eine Variation der Messmethode den Wert gezielt beeinflussen und damit die KSLL unterlaufen könne.

„Weil die Genauigkeit, die diese Leitlinie im Prinzip fordert, 30 versus 35 Prozent in der Realität so gar nicht zu erbringen ist. Und das ist immer für eine bindende Vorschrift ganz schlecht. Also und das führt dann im schlimmsten Fall nur zu Streitereien“ (TN15; 44).

\subsubsection{Akzeptanz}

Verschiedene Faktoren wurden genannt, die die Akzeptanz solcher expliziten Rationierungsinstrumente beeinflussen könnten. So sei die Kommunikation der KSLL an die Patienten ein wichtiger Aspekt, der in der Entwicklung von KSLL mitgedacht werden müsse und der einen Vorteil gegenüber einer impliziten Rationierung darstelle. Eine solche Kommunikation wird als Teil der Aufklärung der Patienten gesehen, also einem transparenten Umgang der Ärzte mit einem Vorgehen nach expliziten Regeln.

Durch eine offene Information der Patienten bezüglich Nutzen, aber auch Kosten medizinischer Behandlungen, könnten diese ein Bewusstsein dafür entwickeln. Voraussetzung für ein Verständnis der Kostendimension sei also eine Aufklärung über den Nutzen medizinischer Behandlungen. Solch ein Kosten- und Nutzenbewusstsein betrifft jedoch nicht nur die Patienten (und damit die potenziell Betroffenen), sondern gleichermaßen die Ärzte. Praktisch könnte das bedeuten, dass solche Rationierungsinstrumente in verschiedenen Versionen erstellt werden, um die Verständlichkeit für Patienten zu erhöhen.

„Und deswegen kann man die Leute nur aufklären, was vorhin erwähnt, also dass man ganz massiv die Patienten auch selber mit ins Boot nimmt und das Bewusstsein einfach schafft" (TN10; 112).

„Ich denke, dass kostensensible Leitlinien eben halt auch in der Bevölkerung kommuniziert werden müssen, um das Angebot klarzumachen, die Kosten klarzumachen und auch dem Einzelnen klarzumachen: was kostet denn was eigentlich?“ (TN7; 124).

Eine weitere wichtige Voraussetzung für die Akzeptanz betrifft die Frage, wessen Interessen mit den Leitlinien verbunden sind. Fachgesellschaften sollten an der Erstellung beteiligt sein, um eine Akzeptanz seitens der Leistungserbrin- 
ger zu gewährleisten. Wer konkret Leitlinien erstellt ist weniger wichtig, als dass alle relevanten Interessen berücksichtigt werden. Wenn deutlich würde, dass die Erstellung solcher Leitlinien nicht durch politische Interessen motiviert ist und die Initiatoren eher unabhängige Institutionen sind, sei eher eine Akzeptanz der KSLL zu erwarten.

„Wobei wie gesagt die Argumentation mit der Kosteneffektivität muss man sehr genau aufpassen, wer die für sich anführt“"(TN12;30).

„Also auf der Ebene des einzelnen Arztes ... Patient, würde ich sagen: da hat man, glaube ich, schon verloren. Man muss das vorher sozusagen eingelocht haben gesellschaftlich, dass es akzeptiert ist, ..." (TN19; 40).

Weiterhin erhöhe sich die Akzeptanz, wenn durch die Beachtung der Kosten auch die Qualität der Behandlung erhöht würde. Der Patient könne möglicherweise auch einen individuellen Vorteil durch die Nichtbehandlung erleben, da die mit der Behandlung verbundenen Risiken mittelbar auch die Lebensqualität einschränken könnten. Die Wahrnehmung medizinischer Vorteile könnte das Gefühl reduzieren, dass an ihm nur gespart würde. Somit stehe die Behandlung nach KSLL einer Erhöhung der Behandlungsqualität nicht grundsätzlich entgegen.

„... sehr starker Eingriff, wenn man so einen Defibrillator reingesetzt bekommt, das ist ja im Grunde das ... man kann jetzt also auf der einen Seite von einem geringeren Nutzen sprechen, man kann aber natürlich auch auf der anderen Seite den Schaden, den so ein Eingriff bedeutet und die Eingriffstiefe und die Belastung, die das für den Patienten darstellt, auch stark machen, wenn man ihm das erläutert, warum es bei ihm nicht sinnvoll ist, das einzusetzen“"(TN2;30).

Als Hemmnisse und Schwierigkeiten wurden politische Rationalitäten genannt, die durch eine Scheu gegenüber Kosten-Argumentationsfiguren gekennzeichnet seien. Bei solcherart Kostenentscheidungen sei eine Politikferne eher erwünscht, da sich der Politik das Problem des Denkens und Handelns in Wahlperioden und der möglichen Wählerverluste stellte. Auch sei politisch kein Gewinn zu erwarten, wenn das Thema der Kosten-Nutzen-Entscheidung öffentlich auf die Agenda käme. Explizite Regelungen einer Rationierung seien politisch eine tabuisierte Diskussion. Auch hier werden Interessenkonflikte angesprochen und auf die Vorsicht hingewiesen, wer solche Kosteneffektivitätsdiskussionen führt und welche Ziele damit verbunden sind. Selbst wenn eine Legitimation vorhanden sei, würde sich keiner trauen, eine solche Diskussion anzustoßen, zu führen und auch auszuhalten. Gerade die Debatte um Schwellenwerte sollte aus diesem Grund vom Parlament geführt werden.

„... aber auch die Politik scheut sich ja wie eben der berühmte Teufel das berühmte Weihwasser mit so einer Argumentationsfigur bestimmte Ausrichtungen von Versorgung zu vertreten" (TN12;30). 
9 Kostensensible Leitlinien: Einschätzungen von Entscheidungsträgern und Interessenvertretern im deutschen Gesundheitswesen

Auf ärztlicher Seite sei eine geringere Akzeptanz zu erwarten, wenn die explizite Rationierung vor allem Leistungen beinhalte, die für die Klinik oder die Praxis finanzielle Gewinne einbrächten. Auch stünde der Rationierung das Behandlungskonzept von Ärzten gegenüber, dass jeder Patient alle medizinischen Leistungen erhalten sollte.

„Wenn es möglich ist, dass sozusagen über die für das Cesamtsystem weniger effiziente Anwendungsweise insgesamt mehr Geld verdient wird, dann wird es auch schwierig sein. Ich glaube, insofern wird die Akzeptanz eben nicht nur von den Inhalten, sondern auch vom Umfeld abhängen " $(\mathrm{TN1} ; 32)$.

Aus Sicht der Versicherten könne sich der Aspekt der Betroffenheit erheblich auf die Akzeptanz auswirken: zwar sei eine prinzipielle Zustimmung vorstellbar, wenn es jedoch um den speziellen Patienten ginge, könne die Akzeptanz sinken.

„Vor allen Dingen die Tatsache, dass ich es nicht kriege, ist für mich wichtiger, weil ich die entgangene Chance sehr hoch werte, als eben die Wahrscheinlichkeit, dass es mir vielleicht nicht geholfen hätte, wenn ich es denn bekommen hätte. Und das ist genau die Diskussion, die in den Medien ja wunderbar dargestellt werden kann“ $(\mathrm{TN} 1 ; 64)$

„Das zweite Problem sehe ich in der Vermittelbarkeit, weil wir in Deutschland eine Kultur haben, dass wir es sehr leicht uns machen, kollektive Entscheidungen mit Härte für alle zu treffen aber die individuell möglichst nicht auf einen selber anzuwenden“ (TN13; 11).

\subsubsection{Praktikabilität}

Eng verbunden mit methodischen Gesichtspunkten stellen sich Fragen der Umsetzung: Welche Bedingungen müssten gegeben sein, damit die KSLL im klinischen Alltag auch praktikabel sind? Zum einen spielen Anreizsysteme eine wichtige Rolle, um den konsequenten Einsatz von KSLL zu gewährleisten. Diejenigen, die für die Einführung solcher kostenberücksichtigenden Leitlinien verantwortlich seien, müssten Monitoring- und Feedbacksysteme schaffen, die eine gewisse Kontrolle ermöglichen, aber auch dem einzelnen Arzt die Möglichkeit geben, nach diesen Leitlinien zu handeln, und implizite Formen der Rationierung überflüssig machen. Als Anreize für Leistungserbringer seien bestimmte Honorierungen (wie Bonuszahlung bei Anwendung oder reduzierte Vergütung bei Nichtanwendung) denkbar. Bei Nichtanwendung sei eine verpflichtende Erklärung der Leistungserbringer vorstellbar, die eine Rechtfertigung beinhaltet. Hier ergeben sich Berührungspunkte zur Verbindlichkeit der KSLL, die später noch ausführlicher diskutiert wird.

„Vorausgesetzt, es gibt ein akzeptiertes Verfahren, könnte ich mir sehr gut vorstellen, dass das dazu beiträgt, insbesondere kostenaufwendige Verfahren in wesentlich konzentrierterer Weise zu verwenden, wenn denn auch ein irgendwie gearteter Anreiz oder ein Monitoring besteht“ ( $\mathrm{TN} \mathrm{i} ; 28)$. 
Das Format der Leitlinie sei ein weiterer wichtiger Aspekt bei der Umsetzung. Wenn die Kriterien für die klinische Praxis auch trennscharf seien und eine klare Entscheidung ermöglichen, könnten sie eine Anwendung erfahren. Dabei sei ein sofortiges Verstehen dieser Leitlinie von Nöten, was auf die Plausibilität und auch die Kürze der Darstellung solcher Behandlungsleitlinien verweist.

„Wenn Sie es schaffen sozusagen so eine Leitlinie mithilfe der Darstellung der Empfehlungen auch unter Berücksichtigung der Kosten oder der Kosteneffektivität in so ein praktisches Format zu bringen, was sofort intuitiv verständlich ist, dann haben Sie sicherlich ein dickes Plus“ (TN3; 32).

\subsubsection{Arzt-Patient-Beziehung}

Die Befragten erwarten unterschiedliche Einflüsse des Einsatzes expliziter Leitlinien auf die Beziehung zwischen Arzt und Patient. Eine Entlastung von Verantwortung für Zuteilungsentscheidungen durch ein solches explizites Instrument stelle für den Arzt eine Erleichterung dar. Dabei spiele die kommunikative Vermittlung an den Patienten eine entscheidende Rolle, da durch eine Transparenz die Beziehung zwischen Patient und Arzt keinen Schaden nehme, möglicherweise sogar gestärkt werden könne. In einer strikt definierten Regelleistungssituation im ambulanten Bereich könnten diese Leitlinien die Behandlungssituation zwischen Arzt und Patient erheblich verbessern.

„Das wäre mit Sicherheit eine Entlastung, wenn man in eine Patientenakte schreiben könnte nach Protokoll X,Y,Z und kostensensibler Leitlinie kein Fall für ..." (TN13; 19).

Durch explizite Vorgaben könne dem momentan erlebten Konkurrenzkampf zwischen Leistungsanbietern Einhalt geboten werden, was gleichfalls zu einer Verbesserung der ärztlichen Situation beitrage.

„... wenn man da entsprechend Leitlinien setzt: pass auf, das ist nicht besser und in dem und dem Punkt sind sie ... ist es bewiesen, dass es gut ist aber dann darf es auch jeder einsetzen und darfesjeder haben, dann ist der Konkurrenzkampfweg“(TN8; 46).

Weiterhin gäbe es bei einer expliziten Regelung auch haftungsrechtliche Erleichterungen für Ärzte, da ihnen eine Unsicherheit in Entscheidungen abgenommen würde und sie diese vor dem Patienten anhand expliziter Vorgaben rechtfertigen können.

„Wenn er es nicht in Ordnung findet, hat er was explizites, an dem er sich reiben kann und dann kann er in Deutschland einfach dem Patienten sagen: es gibt da so eine Leitlinie und ich bin damit aber überhaupt nicht einverstanden, also so sie werden hier in ihren Möglichkeiten beschnitten, gehen sie zum Sozialgericht und das Sozialgericht wird dem Patienten sofort recht geben" (TN14; 38).

„Aber Sie wissen, dass die Ärzte ja vor dem Haftungsrecht noch mehr Angst haben, als vor dem Sozialrecht“"(TN13; 122). 
9 Kostensensible Leitlinien: Einschätzungen von Entscheidungsträgern und Interessenvertretern im deutschen Gesundheitswesen

Dem beruflichen Ethos der Ärzte und dem damit verbundenen Anspruch nach einer umfassenden Behandlung des Patienten, könnten die kostensensiblen Einschränkungen gegenüber stehen. Der resultierende Gewissenskonflikt würde keine Erleichterung bedeuten, sondern vielmehr die Ärzte anhaltend belasten. Die KSLL stellten ein weiteres Instrument dar, um Ärzte in ihrer Entscheidungsfreiheit einzuschränken, und könnten somit möglicherweise eine Kostendiskussion auf die Patienten verlagern. Der derzeitige Status quo würde somit erhalten bleiben und für die Arzt-Patient-Beziehung ließen sich keine Vorteile durch den Einsatz der KSLL ausmachen.

„Grundsätzlich ist es für einen Arzt natürlich eine Belastung, weil er immer alles tun will für den Patienten. Das ist ja klar. Wenn ihm dann einer sagt: du darfst das und das aus den und den Gründen nur noch bis zu einem bestimmten Punkt machen, ist es schon mal eine Belastung" (TNg; 50).

Parallel dazu könnte sich der momentan schon bestehende Konkurrenzkampf unter Kliniken und Versicherern eher verschärfen, wobei der Arzt zwischen Patient und Kostenträger stünde und einen Doppelkonflikt zu tragen hätte; auch in diesem Bereich sei dann keine Veränderung der aktuellen Situation für die Ärzte anzunehmen.

\subsubsection{Verbindlichkeit}

Die Einschätzung der Verbindlichkeit ist verbunden mit der Frage, ob und vor allem nach welchen Kriterien Ausnahmen möglich sein sollten. Da es sich bei den KSLL um Leitlinien und nicht um Richtlinien handelt, wird eine ausnahmslose Verbindlichkeit nicht befürwortet. Dabei stellt sich die Frage, inwieweit eine solche Leitlinie durch Ausnahmeregelungen in ihrer effektiven Anwendung nicht unterhöhlt würde. Hoch könne die Verbindlichkeit vor allem im Leistungsbereich der Gesetzlichen Krankenversicherung sein, also wenn es um eine mögliche Basisversicherung ginge.

„Leitlinien sind streng genommen natürlich rechtlich nicht verbindlich. Deswegen sind es ja Leitlinien und keine Richtlinien. Und für kostensensible Leitlinien istes halt so, dass natürlich ... da müsste man praktisch den medizinischen und den ökonomischen Teil voneinander abtrennen" (TN3; 26).

Die Entscheidungsautonomie des Arztes müsse als Letztentscheidung jedoch gewährleistet bleiben, wobei klinische Erfahrung, aber auch die individuelle Gesamtsituation des Patienten zu berücksichtigen seien. Solcherart Ausnahmen sollten innerhalb des Monitoringsystems transparent gemacht werden und begründbar sein. Eine Vorabfestlegung möglicher Ausnahmekriterien würde die Transparenz erhöhen und willkürlichen Abweichungen entgegenwirken. Da eine ausreichende Basisversorgung damit vorhanden wäre und die Möglichkeit einer privaten Zusatzversicherung bestünde, stelle dies ein gerechtes Verfahren dar. 
„Nach welchen Kriterien kann man abweichen, das ist natürlich die Frage. Es würden ja nur, denke ich ... ich sehe im Moment jetzt nur medizinische. Ich sehe jetzt nicht unbedingt, dass man sagen würde: aus Kostengründen müsste ... würde der Doktor da abweichen. Ich würde tatsächlich nur medizinische Argumente gelten lassen dann“ (TN17; 22).

Ausgehend von angenommenen Schwierigkeiten in der Umsetzung, aber auch Einschränkungen bei der Akzeptanz, wird die Verbindlichkeit eines solchen Instrumentes als gering gesehen. Vorstellbar sei, dass die KSLL als entscheidungsbegleitend, jedoch nicht als entscheidungsleitend eingesetzt würden.
„Man müsste das eher eben als Ergänzung der medizinischen Entscheidungskriterien formulieren in Bezug auf die konkrete Patientengruppe mit dem gleichen Outcome- Ziel und sagen: wenn ihr da jetzt abwägen könntet, dann könntet ihr mit den und den Indikationen bei der Erkrankung am meisten mit euren gegebenen Mitteln erreichen. Ich glaube, das ist eine interessante Perspektive. Ob sie in der Praxis erzwungen wer- den kann als entscheidungsrelevant, das wage ich zu bezweifeln“ (TN1; 52).

\subsubsection{Funktion der Kostensensiblen Leitlinien}

Die Erleichterung für die Arzt-Patient-Beziehung stellt eine mögliche Funktion der KSLL dar; auf einer übergeordneten Ebene wären weitere Funktionen denkbar. So könnten KSLL auch zu einem rationalen Ressourcenmanagement beitragen, da sie eine Zielbeschreibung ermöglichen, das Prinzip der Rationierung verdeutlichen und somit hilfreich für Priorisierungsentscheidungen sein könnten. Dabei müsse Transparenz geschaffen werden über Konsequenzen von Entscheidungen, was durch eine offene Kommunikation dieser expliziten Regelungen möglich sei. Es könne eine Signalwirkung entstehen, die sich auf das Bewusstsein des Nutzens und der Kosten medizinischer Behandlung beziehe. Als Ergebnis sei eine Erhöhung der Qualität der Behandlung denkbar.

„In dem Moment, wo Sie so eine Schwelle als öffentlich ohne einer Diskriminierung oder ohne in Verruf zu geraten anführen können, in dem Moment ist es natürlich ein rationales Konstrukt, was dann auch zumindest die Annährung an so eine Schwelle transparenter machen würde und wäre dann sicherlich allemal ein Stück weit ehrlicher, wenn man so was hat, als wenn man gar nichts hat" (TN12;38).

„Das ist ja eigentlich genau das, was man heute von einem vernünftigen Versorgungsmanagement erwarten würde, insofern ist das eigentlich etwas, was nachvollziehbar ist und in die richtige Richtung geht" (TN12; 16).

Bei der Definition des Leistungsumfangs der Gesetzlichen Krankenversicherung könnten KSLL einer Grenzziehung des Mitteleinsatzes dienen. Medizinisch Notwendiges ließe sich damit gut als Basisversicherung beschreiben und von anderen Leistungen abgrenzen, die in privaten Versicherungen zusätzlich aufgenommen werden könnten. 
9 Kostensensible Leitlinien: Einschätzungen von Entscheidungsträgern und Interessenvertretern im deutschen Gesundheitswesen

„Natürlich da Leitlinien, Kostenleitlinien, sage ich mal so, blöder Ausdruck aber ich weiß keinen besseren, würden da denke schon, um diese Gradwanderung, um diese Grenze Basis und wann fängt der Gesundheitsmarkt an, die wären da genau der Schlüssel dazu“ (TN6; 95).

Hervorgehoben wurde der Beitrag der KSLL zu einer als notwendig empfundenen Diskussion über Rationierung in Deutschland. Dabei sei es wichtig, überhaupt in die öffentliche Debatte einzusteigen, wozu die KSLL einen erheblichen Anstoß leisten könnten.

„Ich glaube auch, dass diese Leitlinien als Diskussionsbeitrag hilfreich sein können. Insofern möchte ich Sie eigentlich ermutigen, da eher weiterzumachen, auch wenn ich insgesamt sehr sehr kritisch geurteilt habe“ (TN1; 68).

\subsubsection{Ersteller der Leitlinien}

Die Frage nach den Initiatoren und Erstellern solcher Kostensensiblen Leitlinien wurde sehr unterschiedlich beantwortet. Zum einen stelle der Konsens zwischen allen Beteiligten eine wichtige Voraussetzung für die Akzeptanz dar, jedoch könne dabei nicht ausgeschlossen werden, dass bestimmte Interessensgruppen ihre Ziele durchsetzen bzw. diese dann dominieren. Ein Gremium aus medizinischen, juristischen, ökonomischen und ethischen Sachverständigen mit Beteiligung von Patientenvertretern wurde als größtmöglicher Expertenkreis genannt. Die Beteiligung von Fachgesellschaften einer anderen Spezifizierung als der die jeweilige Leitlinie betreffenden, könne Interessenkonflikte reduzieren.

„Also ich würde mal sagen, ein paritätisch besetztes Gremium aus allen, die die nachher dann auch vertreten umsetzen müssen. Ja“ (TN19; 56).

Auch sei ein Stufenverfahren bei der Erstellung denkbar, bei dem die jeweiligen Sachverständigen einzelne Komponenten erarbeiten, die dann in einem letzten Schritt zusammengeführt werden können. Dabei wird auf die vorhandenen Institutionen, wie den G-BA verwiesen, der bereits mit der Erstellung von Leitlinien betraut sei; für die KSLL sei keine neue Institution notwendig.

„In unserem System, wie wir es heute noch kennen, ... Wird das so ein Institut wie Gem-BA sein. Ich finde am Gem-BA interessant, dass er versucht, ganz viele Leute mit einzubinden. Da das dann natürlich wieder so viele sind, bedient er sich dann sofort wieder einer Maschinerie, die dann sehr einseitig gefärbt ist, wie IQWIG oder ähnlichem, um dort dann wieder sozusagen sich die Entscheidung ja mundgerecht vorbereiten zu lassen. Sein wir doch auch ehrlich, IQWIG legt einen Entscheidungsvorschlag vor und der wird im Gem-BA abgesegnet. Das heißt, hier muss man dann wieder aufpassen, dass es nicht ein bisschen auf die eine Seite zu stark gewichtet wird“"(TN13;37). 


\subsection{Diskussion}

Die befragten Entscheidungsträger und Interessenvertreter aus dem deutschen Gesundheitswesen konnten sich anhand der beiden exemplarischen Kostensensiblen Leitlinien (KSLL) für Medikamente-freisetzende Stents (DES) und für implantierbare Kardioverter-Defibrillatoren (ICD) ein Bild davon machen, wie eine explizite Rationierung in zwei Anwendungsbereichen - der Koronaren Herzerkrankung und der Herzinsuffizienz - konkret aussehen könnte. Eine breitere Zustimmung erfuhr das hinter den KSLL stehende Konzept einer expliziten Rationierung nach klar definierten Regeln. Insbesondere die evidenzbasierte Abschätzung von Wirksamkeit und Nutzen und die Identifizierung von Patientensubgruppen wurden von den Interviewteilnehmern positiv beurteilt. Der transparente Entscheidungsprozess macht die Abwägung von Kosten und Nutzen offensichtlich, was die Akzeptanz der resultierenden Zuteilungsentscheidungen fördern würde. Diese Einschätzungen decken sich mit den Urteilen der von uns befragten Ärzte (Strech et al. 2009b). Diese lobten die Transparenz der Entscheidungsfindung und fanden es insbesondere in den Situationen hilfreich, eine explizite Orientierung durch die KSLL zu haben, wenn verschiedene kostenintensive Alternativen zur Verfügung stehen. Auf Crundlage der KSLL könnten sie sich besser für die Entscheidungen rechtfertigen, sowohl vor dem klinikinternen Controlling als auch gegenüber den Patienten.

Diese Befunde werden durch die Ergebnisse internationaler sozialempirischer Forschung mit Ärzten im stationären und ambulanten Bereich gestützt (Strech et al. 2008b). Diese liefern verschiedene Argumente für die Anwendung expliziter Instrumente der Leistungssteuerung. Da bei den KSLL die Auswirkungen der Entscheidungen auf Qualität und Kosten der Versorgung evidenzbasiert abgewogen werden können, lässt sich die Qualität der Versorgung unter Bedingungen der Ressourcenknappheit besser sichern, als bei den sonst vorherrschenden impliziten Rationierungen. Dort dominieren medizinisch wenig rationale Strategien wie Verlegung, frühzeitige Entlassung oder längere Wartezeiten (Hurst et al. 2005). Ärzte äußern immer wieder den Wunsch nach Unterstützung bei Entscheidungen, die Kostengesichtspunkte berücksichtigen (müssen), da sie selbst über unzureichende Kompetenz in diesem Bereich verfügen und mit den Entscheidungen allein gelassen werden - was zu moralischem Stress führen kann (Kalvemark et al. 2004). Evidenzbasierte KSLL, die neben den Nutzendaten auch die Kosten der Maßnahmen berücksichtigen, könnten in diesen Situationen eine wertvolle Entscheidungsunterstützung darstellen und die Ärzte in ihrer Verantwortung entlasten.

Trotz dieser Vorzüge äußerten sich die von uns in der vorliegenden Studie befragten Entscheidungsträger und Interessensvertreter skeptisch, ob die KSLL tatsächlich im deutschen Gesundheitswesen Akzeptanz finden würden. Die Kritik richtete sich dabei vor allem gegen die Grundannahmen der KSLL. Zum einen wurde infrage gestellt, ob im Bereich der GKV überhaupt rationiert wer- 
9 Kostensensible Leitlinien: Einschätzungen von Entscheidungsträgern und Interessenvertretern im deutschen Gesundheitswesen

den müsse. Bevor man aus Kostengründen auf für die Patienten nützliche Leistungen verzichtet, sollten alternative Einsparpotenziale genutzt werden, wie z.B. hohe Gewinne bei bestimmten Leistungserbringern zu reduzieren und durch strukturelle Reformen die Effizienz der Versorgung zu steigern. Konzeptionell trifft dieses Argument sicher zu: Solange es noch Rationalisierungsreserven im System gibt, ist es eigentlich ethisch unvertretbar, Patienten im Rahmen von Rationierungen nützliche Leistungen vorzuenthalten. So plausibel diese Argumentation auf den ersten Blick auch erscheinen mag, so geht sie doch an der Realität des Gesundheitswesens vorbei. Es wird niemals ein vollkommen effizientes System geben, sondern immer eine Koexistenz zwischen Über- und Fehlversorgung einerseits und (impliziten) Rationierungen andererseits. Vor allem wenn letztere vulnerable Patientengruppen benachteiligen (Brockmann 2002), dürfen sie nicht unberücksichtigt bleiben. Wie wir an anderer Stelle ausgeführt haben (Strech und Marckmann 2010), sollten allerdings die Bemühungen um die Schaffung und Aufarbeitung verlässlicher Evidenz zu Nutzen- und Schadenspotenzialen medizinischer Maßnahmen Vorrang haben. Dies erscheint auch aus dem Grunde sinnvoll, weil alle medizinisch, ethisch und ökonomisch vertretbaren Bemühungen zur Priorisierung oder Rationierung verlässliche Informationen zu Nutzen und Schaden voraussetzen - wie z.B. auch die hier diskutierten KSLL. Entscheidend für die Legitimität von Rationierungsbemühungen ist dann, dass gleichzeitig ausreichende Bemühungen zur Eliminierung von Ineffizienzen unternommen werden (vgl. hierzu das Konzept der „benchmarks of efficiency“ von [Strech und Danis 2014]).

Weitere Skepsis der Studienteilnehmer bezog sich auf die Voraussetzungen im deutschen Gesundheitssystem bzw. in der deutschen Gesellschaft. Bislang sei keine Bereitschaft vorhanden - und dies trifft insbesondere auf die deutsche Gesundheitspolitik zu -, offen über Grenzen der solidarisch finanzierten Gesundheitsversorgung zu sprechen. Nach wie vor dominerte in der Bevölkerung die Auffassung, genährt von den Versprechungen der Politik, dass alle medizinisch sinnvollen Leistungen unabhängig von den Kosten von der GKV finanziert werden. Zudem würde ein Ausschluss von medizinischen Maßnahmen für bestimmte Patientensubgruppen zu einer Zweiklassenmedizin führen, da ein privater Zukauf der von der GKV nicht bezahlten Leistungen möglich sei und von den sozioökonomisch besser gestellten Teilen der Bevölkerung sicher auch praktiziert werde. Dies widerspreche den Crundannahmen des öffentlichen deutschen Krankenversicherungssystems. Diese Auffassung ignoriert die Tatsache, dass es aktuell bereits Rationierungen im System gibt, wie u.a. unsere Studie im Forschungsverbund "Allokation“ belegt (Strech et al. 2009a) (s. Kap. 1). Diese Rationierungen laufen intransparent, nach wechselnden, ethisch häufig nicht gut begründeten Kriterien ab und drohen vor allem vulnerable Patientengruppen zu benachteiligen. Vor diesem Hintergrund könnte eine kontrollierte, evidenzbasierte Rationierung mittels Kostensensibler Leitlinien das geringere Übel sein, da über alle Patientengruppen hinweg nur 
dort Leistungen ausgeschlossen werden, wo die Patienten nur einen geringen Zusatznutzen (bei hohen Zusatzkosten) von den Maßnahmen haben. Zu berücksichtigen ist in diesem Zusammenhang zudem, dass es bereits heute z.T. erhebliche Unterschiede in der Versorgung zwischen privat und gesetzlich versicherten Patienten gibt.

Dennoch ist die Skepsis der Befragten in diesem Punkt sehr ernst zu nehmen. Ansätze einer expliziten Leistungssteuerung im Gesundheitswesen wie die KSLL werden sich in einem demokratischen Rechtsstaat nur dann erfolgreich implementieren lassen, wenn es ausreichend Rückhalt in der Bevölkerung gibt. Diesen Rückhalt scheint es bislang aber nicht in ausreichender Weise zu geben, sodass die Skepsis der Experten und Interessenvertreter durchaus nachvollziehbar ist. Die internationalen Erfahrungen mit Priorisierungen im Gesundheitswesen stützen diese Einschätzung (Marckmann 2009). Den erfolgreichen Priorisierungsansätzen in Norwegen und insbesondere Schweden (Raspe und Meyer 2009) gingen jeweils die Etablierung einer nationalen Prioritätenkommission und intensive öffentliche Diskussionen voraus, in denen ausdrücklich anerkannt wurde, dass aufgrund der zunehmenden Diskrepanz zwischen medizinisch Möglichem und solidarisch Finanzierbarem Schwerpunkte in der Versorgung gesetzt werden müssen. Der intensive gesellschaftspolitische Bereich bereitete den Boden für konkrete und insbesondere in Schweden weit gediehene Priorisierungsansätze. Zurecht fordert deshalb die Arbeitsgruppe „Priorisierung im Gesundheitswesen“ der BÄK einen intensivierten öffentlich-medialen Diskurs über Priorisierung, idealerweise unterstützt von einem eigens hierfür organisierten Forum wie z.B. durch einen Gesundheitsrat (Raspe und Schulze 2013).

Darüber hinaus liefern die Interviews verschiedene Hinweise auf methodische Herausforderungen bei der Erstellung der KSLL. Insbesondere bereite die oft nicht ausreichende Datenlage zu Effektivität und Nutzen der Maßnahmen Probleme. Hier seien zunächst Verbesserungen erforderlich, bevor die Nutzendaten in den KSLL mit Kostendaten verbunden werden. Diese Einschätzung wurde durch die Erfahrungen bei der Erstellung exemplarischer KSLL in unserem Forschungsverbund Allokation bestätigt. Die ursprünglich auch für den Bereich der Intensivmedizin geplanten KSLL konnten vor allem aufgrund der schlechten Evidenzlage mit einer unzureichenden oder widersprüchlichen Identifizierung von Subgruppen mit unterschiedlichem Nutzengewinn nicht erstellt werden. Dies scheint aber nicht generell gegen das Konzept der KSLL zu sprechen, sondern grenzt die mögliche Anwendung auf die Versorgungsbereiche ein, in denen die Evidenzlage zu Nutzen- und Schadenspotenzialen der Maßnahmen vergleichsweise gut ist. Die Verwendung der qualitätskorrigierten Lebensjahre als generisches Nutzenmaß sahen viele Studienteilnehmer ebenfalls kritisch, da das Konzept in Deutschland bislang keinen ausreichenden Rückhalt habe. 
9 Kostensensible Leitlinien: Einschätzungen von Entscheidungsträgern und Interessenvertretern im deutschen Gesundheitswesen

Als eine besondere methodische Herausforderung der KSLL identifizierten die Interviewpartner die Frage der Grenzwert-Festsetzung. Wenn bei der Erarbeitung der KSLL Patientensubgruppen definiert werden, die jeweils einen unterschiedlichen Zusatznutzen und damit bei gleichbleibenden Kosten auch eine unterschiedliche Kosteneffektivität durch die Maßnahme aufweisen, stellt sich die Frage, bis zu welchem Kosten-Nutzen-Verhältnis die betreffende Maßnahme den Patienten zukommen soll bzw. ab welchem Grenzwert die Patienten auf eine alternative Maßnahme ausweichen sollen. Zu Recht weisen die Interviewteilnehmer darauf hin, dass sich diese Grenzwerte nicht rein wissenschaftlich begründen lassen, sondern auf Werturteilen beruhen, die letztlich auf die gesellschaftliche Zahlungsbereitschaft verweisen. Zu klären wäre in diesem Zusammenhang auch, ob es einen indikationsübergreifenden Grenzwert für das Kosten-Nutzen-Verhältnis geben soll oder ob indikationsspezifische Grenzwerte gesetzt werden sollen (s. Kap. 2). Da es bislang in Deutschland keine allgemein verbindlichen Vorgaben für ein maximal akzeptables Kosten-Nutzen-Verhältnis gibt, sei die Erstellung der KSLL - so die Interviewteilnehmer - dem Einfluss von Interessengruppen ausgesetzt. Hinzu kommt, dass sich die Subgruppen oft nur mit wenig verlässlichen Untersuchungsmethoden differenzieren lassen (vgl. z.B. die Bestimmung der linksventrikulären Ejektionsfraktion [LVEF] beim Einsatz eines ICDs), was Möglichkeiten für Manipulationen eröffne. Um eine möglichst große Objektivität der KSLL sicherzustellen, sollten die wissenschaftlichen medizinischen Fachgesellschaften in die Erstellung einbezogen werden. Zu befürworten sei auch ein Stufenverfahren, bei dem die Aufarbeitung der wissenschaftlichen Evidenz $\mathrm{zu}$ Kosten und Nutzen der medizinischen Maßnahme von der Entscheidung über die Indikationseinschränkung in den KSLL zu trennen. Letztere sollte von einem Gremium mit medizinischen, juristischen, ökonomischen und ethischen Sachverständigen getroffen werden. Vorgeschlagen wurde hierfür z.B. der Gemeinsame Bundesausschuss (G-BA), der als Gremium der gemeinsamen Selbstverwaltung Entscheidungen über den Leistungskatalog der gesetzlichen Krankenkasse trifft. Diese Trennung von Evidenzaufarbeitung und Erstattungsentscheidung erscheint auch aus ethischer Sicht sinnvoll.

Die erfolgreiche Implementierung der KSLL in der Praxis hängt nach Einschätzung der Interviewteilnehmer von verschiedenen Faktoren ab. Wenn sich zeigen sollte, dass die Anwendung der KSLL auch die Qualität der Versorgung verbessern kann (z.B. durch die Vermeidung von Belastungen und Risiken durch die medizinischen Maßnahmen bei Patientensubgruppen, die nur einen geringen Zusatznutzen gehabt hätten, vgl. z.B. die Interventionsrisiken bei der Implantierung eines ICDs), würde sich das sicher positiv auf die Akzeptanz der KSLL auswirken. Relevant seien darüber die finanziellen Anreize für den Arzt bzw. die Klinik, die einer effektiven Anwendung von KSLL entgegenstehen können, wenn die in der Indikation eingeschränkten Maßnahmen für die Leistungserbringer finanziell lukrativ sind. Die Auswirkungen auf die ArztPatient-Beziehung wurden ambivalent eingeschätzt: Auf der einen Seite wür- 
den die KSLL durch die klar definierten, für alle (gesetzlich versicherten) Patienten gleichermaßen verbindlichen Vorgaben das Verhältnis entlasten können. Leistungseinschränkungen könnten auf Grundlage der KSLL besser gegenüber dem Patienten gerechtfertigt werden. Diese Einschätzungen bestätigen unsere Hypothese, dass explizite Rationierungen mittels KSLL die Arzt-Patient-Beziehung entlasten, weil die Entscheidungen nicht vom Arzt im Einzelfall getroffen werden müssen.

Allerdings wiesen die Studienteilnehmer darauf hin, dass die Anwendung von KSLL auch mit Belastungen verbunden sei, da die Indikationseinschränkungen mit dem ärztlichen Ethos konfligierten. Dies setzt jedoch eine Interpretation des ärztlichen Ethos voraus, nach der der Arzt ausschließlich dem Wohlergehen des individuellen Patienten verpflichtet ist, unabhängig von den dadurch entstehenden Kosten. „Modernere“ Interpretationen des ärztlichen Ethos, wie bspw. die Charta on Medical Professionalism (2002) fordern zwar nach wie vor, die Interessen des Patienten über diejenigen des Arztes zu stellen, betonen aber gleichzeitig die ärztliche Verantwortung für die gerechte Verteilung begrenzter Ressourcen, was auch die Beteiligung an der Erstellung von Leitlinien für eine kosteneffektive Versorgung einschließt. Dies setzt aber voraus, dass sich die Ärzteschaft den Fragen der Verteilung begrenzter Ressourcen im Gesundheitswesen stellt und Perspektiven erarbeitet, wie Ärzte in der Praxis diese Verantwortung wahrnehmen können (Raspe und Schulze 2013).

Trotz aller methodischer Schwierigkeiten und grundsätzlicher Bedenken schreiben die Experten und Interessenvertreter den KSLL eine wichtige Funktion zu, da sie den Diskurs über den Umgang mit begrenzt verfügbaren finanziellen Ressourcen im Gesundheitswesen auf eine sachliche Ebene verlagern und ein rationales, wissenschaftlich fundiertes Instrument zur Diskussion stellen. Insgesamt könnten die KSLL das Bewusstsein über den Zusammenhang zwischen Nutzen und Kosten medizinischer Maßnahmen fördern und damit einen Beitrag zu einem rationalen Ressourcenmanagement leisten. Auch die öffentliche Debatte könnte durch die konkreten Modelle einer expliziten Leistungssteuerung befruchtet werden.

\subsection{Limitationen}

Diese Untersuchung liefert keine repräsentativen Ergebnisse bezüglich Verteilung oder Häufigkeit der von den Interviewpartnern identifizierten Stärken und Schwächen der KSLL. Vielmehr versteht sie sich als Darstellung eines Spektrums möglicher Aspekte, die für die Beurteilung von KSLL als ein Instrument der expliziten Leistungssteuerung relevant sind. Es handelt sich um Einschätzungen von Experten und Interessenvertretern im deutschen Gesundheitswesen, die in unterschiedlicher Weise Verantwortung für die Cestaltung des Systems tragen. Zu ergänzen sind diese Befunde durch Stellungnahmen 
aus Sicht spezifischer, potenziell betroffener Patienten: Stellen die KSLL eine akzeptable Form der Leistungssteuerung dar? Diese Aspekte wurden in einer weiteren Studie untersucht, deren Ergebnisse im folgenden Kapitel 10 präsentiert werden.

\section{Autorenerklärung und Danksagung}

Diese Untersuchung wurde im Rahmen des Forschungsverbunds „Allokation“ mit Mitteln des Bundesministeriums für Bildung und Forschung (BMBF) gefördert (Fkz 01GP0608). Die Verantwortung für den Inhalt dieser Veröffentlichung liegt bei den Autoren. Wir danken den Interviewpartnern für ihre Bereitschaft, uns ihre Zeit und ihr Wissen für diese Studie zur Verfügung zu stellen. Hendrik Bittorf sei für die Unterstützung bei der Transkription gedankt.

\section{Literatur}

Boldt J, Schöllhorn T (2008) Rationierung ist längst Realität. Ergebnisse einer Fragebogenaktion auf deutschen Intensivstationen. Deutsches Ärzteblatt 105, A995-7

Brockmann $\mathrm{H}$ (2002) Why is less money spent on health care for the elderly than for the rest of the population? Health care rationing in German hospitals. Soc Sci Med 55, 593-608

Hurst SA, Hull SC, DuVal G, Danis M (2005) Physicians' responses to resource constraints. Arch Intern Med 165 , 639-44

Kalvemark S, Hoglund AT, Hansson MG, Westerholm P, Arnetz B (2004) Living with conflicts-ethical dilemmas and moral distress in the health care system. Soc Sci Med 58, 1075-84

Kern A0, Beske F, Lescow H (1999) Leistungseinschränkung oder Rationierung im Gesundheitswesen? Deutsches Ärzteblatt 96, A-113-117

Marckmann G (2007) Zwischen Skylla und Charybdis: Reformoptionen im Gesundheitswesen aus ethischer Perspektive. Gesundheitsökonomie \& Qualitätsmanagement 12, 96-100

Marckmann G (2009) Priorisierung im Gesundheitswesen: Was können wir aus den internationalen Erfahrungen lernen? Z Evid Fortbild Qual Gesundhwes 103, 85-91

Mayring P (2008) Qualitative Inhaltsanalyse. Grundlagen und Techniken. Beltz Verlag Weinheim, Basel

Medical Professionalism Project (2002) Medical professionalism in the new millennium: a physicians' charter. Lancet 359, 520-2

Peters E, Pritzkuleit R, Beske F, Katalinic A (2010) Demografischer Wandel und Krankheitshäufigkeiten: Eine Projektion bis 2050. Bundesgesundheitsblatt Gesundheitsforschung Gesundheitsschutz 53, 417-26

Przyborski A, Wohlrab-Sahr M (2008) Qualitative Sozialforschung. Ein Arbeitsbuch. Oldenbourg Wissenschaftsverlag München

Raspe H, Meyer T (2009) Priorisierung: Vom schwedischen Vorbild lernen. Deutsches Ärzteblatt 106, A 1036-9

Raspe H, Schulze I (2013) Medizinische Versorgung: Ärztlich unterstützte Priorisierung ist notwendig und hilfreich. Deutsches Ärzteblatt 110, A1091-1096

Strech D, Börchers K, Freyer D, Neumann A, Wasem J, Marckmann G (2008a) Ärztliches Handeln bei Mittelknappheit. Ergebnisse einer qualitativen Interviewstudie. Ethik in der Medizin 20, 94-109

Strech D, Danis M (2014) How can bedside rationing be justified despite coexisting inefficiency? The need for 'benchmarks of efficiency'. I Med Ethics 40, 89-93

Strech D, Danis M, Löb M, Marckmann G (2009a) Ausmaß und Auswirkungen von Rationierung in deutschen Krankenhäusern. Ärztliche Einschätzungen aus einer repräsentativen Umfrage. Deutsche Medizinische Wochenschrift 134, 1261-1266 
Strech D, Freyer D, Börchers K, Neumann A, Wasem I, Krukemeyer M G, Marckmann G (2009b) Herausforderungen expliziter Leistungsbegrenzungen durch kostensensible Leitlinien. Ergebnisse einer qualitativen Interviewstudie mit leitenden Klinikärzten. Gesundheitsökonomie \& Qualitätsmanagement 14, 38-43

Strech D, Marckmann G (2010) Wird in deutschen Kliniken rationiert oder nicht? Wie genau wir es wissen und warum es nicht die wichtigste Frage sein sollte. Dtsch Med Wochenschr 135, 1498-502

Strech D, Synofzik M, Marckmann G (2008b) How physicians allocate scarce resources at the bedside: a systematic review of qualitative studies. I Med Philos 33, 80-99

Zentrale Kommission zur Wahrung ethischer Grundsätze in der Medizin und ihren Grenzgebieten (Zentrale Ethikkommission) bei der Bundesärztekammer (2007) Priorisierung medizinischer Leistungen im System der Gesetzlichen Krankenversicherung (GKV). Deutsches Ärzteblatt 104, A-2750-2754 